\title{
A Discrete-Time Technique for the Steady-state Analysis of Nonlinear Class E Amplifiers
}

\author{
F. del-Águila-López, P. Palà-Schönwälder, Member, IEEE, P. Molina-Gaudó, Senior Member and \\ A. Mediano-Heredia, Senior Member, IEEE
}

\begin{abstract}
Switched circuits are widely used, particularly for power electronic applications in which efficiency is important. Of these applications, the class-E amplifier has been given particular attention, since it is theoretically a $\mathbf{1 0 0 \%}$ efficient switched circuit that has been successfully demonstrated in applications such as ballasts, converters, frequency multipliers and communication amplifiers at frequencies as high as $10 \mathrm{GHz}$. However, with increasing power or frequency, nonlinearities become extremely important, for instance, in order to achieve actual class $E$ operation and even to avoid destruction of the switching device.

In this paper, a new method for determining the steady-state response of nonlinear circuits containing ideal switches is proposed. While the method is more general, the description is based on the Class $E$ amplifier because of its inherent interest. The method is based on a time-domain Gear discretization of the circuit equations. A technique for determining the initial samples of the discretized equation of each topology is developed, based on the fact that state variables are constant during switching. Finally, assuming a periodic steady-state, a single algebraic system of nonlinear equations is obtained in which the unknowns are the samples of the control variable of the nonlinearity in the whole signal period. To validate the method described, a comparison with PSpice simulations is provided.
\end{abstract}

Index Terms - Class E, steady-state, discrete-time, nonlinear circuits, switched circuits, circuit analysis.

\section{INTRODUCTION}

$\mathrm{S}$ WITCHED circuits have become widely used due to their efficiency in power-supply circuits and RF power amplifiers, among others. Analysis and simulation tools are essential for designers because they allow the performance of circuits to be accurately predicted and optimized. They also enable the required active device specifications to be determined a priori. General nonlinear switched-circuit analysis techniques have been described for this purpose (see [1], [2] and references therein), and specific approximate techniques have also been developed for resonant converters [3]. Several papers have improved certain aspects of

Manuscript received July 25, 2005. This work was supported in part by the Spanish Dirección General de Investigación under Grant TIC2003-02755.

F. del Águila-López and P. Palà-Schönwälder are with the Department of Signal Theory and Communications, Universitat Politècnica de Catalunya, Barcelona 08034, Spain (e-mail: aguila@tsc.upc.edu). simulation, such as [4], in which a state-space modeling technique is introduced for linear circuits, and [5], in which a general nonlinear continuous formulation procedure for largesignal analysis of switching dc-dc converters is presented. Note that most techniques are formulated in the time-domain because of the inherent difficulty of describing temporal dependence in the frequency domain. There are also methods based on the Volterra series that are suitable for softnonlinearities, such as [6].

In many applications, an exact knowledge of the transient behavior is not critical, as the main interest is in the steadystate waveforms [7], [8], [9]. However, switched circuits may cause initial inconsistent conditions [10], which lead to Dirac impulses that are not correctly handled by some techniques [11]. Several techniques for overcoming these problems, including specific switch models [12], have been developed.

In this paper a new method for the steady-state analysis of switched circuits with nonlinear elements is presented, with an emphasis on applying it to the class $E$ amplifier. The value of this novel approach is in the combination of the following advantages:

1) Computational efficiency is increased by the fact that this method obtains the steady-state waveforms of the nonlinear circuit directly, therefore solving an algebraic nonlinear system of equations.

2) In switched network analysis, the discretization order or the integration step, or both, have to be adaptively reduced in order to accurately determine the initial conditions of the next circuit configuration. In the approach described, as a novelty, a fixed discretization order and an integration step are used, resulting in a constant-rate sampling grid. The initial samples required to start the discretized equation in each topology are obtained directly from the samples prior to switching and assuming state variable conservation.

3) The method is especially suitable for nonlinear switched circuits, since time-domain described nonlinearities can be included directly into the formulation without the need for linearization. This is an advantage compared to harmonic balance methods, in which it is difficult to represent the network changes in the frequency domain

P. Molina-Gaudó and A. Mediano-Heredia are with the Department of Electronic Engineering and Communications, Universidad de Zaragoza, Zaragoza 50018, Spain (e-mail: pimolina@unizar.es). 
and repeated transformations between the time and frequency domains are required.

4) The switch is treated as an ideal element with zero switching time, in contrast with other approaches in which it is appropriately remodeled (e.g. [12], [13]).

5) The proposed approach is especially suitable for sensitivity calculations and lends itself easily to optimization, which is the objective of current research.

There are some limitations of the proposed method. First, the state-space description of the circuit needs to be known. Although the method might well be extended to a circuit containing several switches and several nonlinear elements, this paper focuses on a single switch example and specifically on the class E amplifier. Furthermore, this method relies on the value of the state variables being conserved during the switching process. The extension to include several switches and several nonlinearities does not require any new conceptual developments, but is rather involved and therefore not covered here. This and the inclusion of non-consistent initial conditions are described in [14].

\section{THE Class E AMPLIFIER}

Since the class-E concept was first introduced [15], [16] (Fig 1), many papers have been published describing its performance and design criteria. The device is considered to act as a switch, and the stage includes a tuned output network that forces nominal class E switching conditions: zero-voltage and zero-voltage derivative at the instant of turn ON. Some analytical descriptions of this circuit assume an ideal switch that models the switching device, an infinite tuned output network quality factor $\mathrm{Q}$, and an ideal RF choke in the power supply path. Later works consider finite DC-feed inductance, finite tuned output network Q, nonzero active device ONresistance, nonlinear elements (especially the nonlinear output capacitance of the device) and effects of duty cycle [17] and component variations [18]. Note that in this amplification mode there is a capacitance shunting the device. The required value of this capacitance decreases with increasing frequencies, ultimately being provided merely by the device's output capacitance. Thus, the nonlinear nature of this capacitance is highly relevant to the overall performance of the amplifier [19].

In this paper, the switch is modeled as an ideal switch, i.e. a short-circuit in the on state and an open-circuit in the off state. The switch is assumed to be externally controlled. The dutycycle, $D$, representing the ratio between the time the switch is on and the switching period, is not constrained to 0.5 in this analysis as in [20]. The choke is modeled by the inductance, $L_{q}$ and the corresponding DC resistance $R_{L q}$. The nonlinear capacitor shunting the device is described by

$$
C_{S}(v)=C_{j 0}\left(1+\frac{v}{V_{b i}}\right)^{-\gamma}+C_{l}
$$

with the model parameters $C_{j 0}, V_{b i}$ and $\gamma$ as in [21]. Equation (1) may include a linear term $C_{l}$, which is zero in the case of a class $\mathrm{E}$ amplifier in which the shunting capacitance is provided completely by the switching device. However, since nonlinear inductors or capacitors are best described in terms of flux or charge variables, (1) is rewritten as

$$
q_{S}(v)=\frac{1}{1-\gamma} C_{j 0} V_{b i}\left(1+\frac{v}{V_{b i}}\right)^{1-\gamma}+C_{l} v
$$

This circuit is used as a supporting example to describe the technique proposed in this paper. The next section is devoted to the method description and the results are presented in Section 4.

\section{METHOD DESCRIPTION}

The proposed method is an extension of [22-24], in which the circuit is described by a discretized differential equation in terms of the samples of the control variable of the nonlinear element, i.e. $v$ in Fig. 1. In this paper, as in [22-24], the objective is to compute a vector of samples of a given variable of a switched nonlinear circuit in the steady-state. Specifically, samples of the control variable of the nonlinear element are obtained. Any other current or voltage waveforms can be easily derived from these data. However, in contrast to prior work, the approach described here relies on a state-variable formulation of the circuit, which provides the additional information needed for switching, as will be shown.

\section{A. State equations}

The state equations that describe the circuit depicted in Fig. 1 in the on state are

$$
\left[\begin{array}{c}
\dot{i}_{L q}(t) \\
\dot{i}_{L}(t) \\
\dot{v}_{C}(t) \\
\dot{q}(t)
\end{array}\right]=\left[\begin{array}{cccc}
\frac{-R_{L q}}{L q} & 0 & 0 & 0 \\
0 & \frac{-R}{L} & \frac{-1}{L} & 0 \\
0 & \frac{1}{C} & 0 & 0 \\
1 & -1 & 0 & 0
\end{array}\right]\left[\begin{array}{c}
i_{L q}(t) \\
i_{L}(t) \\
v_{C}(t) \\
q(t)
\end{array}\right]+\left[\begin{array}{c}
\frac{-1}{L q} \\
\frac{1}{L} \\
0 \\
\frac{-1}{R s}
\end{array}\right] v(t)+\left[\begin{array}{c}
\frac{1}{L q} \\
0 \\
0 \\
0
\end{array}\right] v_{g}(t)
$$

and

$$
f(v(t))=\left[\begin{array}{llll}
0 & 0 & 0 & 1
\end{array}\right]\left[\begin{array}{c}
i_{L q}(t) \\
i_{L}(t) \\
v_{C}(t) \\
q(t)
\end{array}\right]+0 \cdot v(t)+0 \cdot v_{g}(t)
$$

where $f(v(t))=q(v(t))$ represents the nonlinear function describing the nonlinear capacitor charge and $v_{g}(t)$ may be any time-varying waveform even though $v_{g}(t)$ is a constant in conventional class $\mathrm{E}$ amplifiers.

Defining

$$
\mathbf{b}=\left\lfloor\begin{array}{llll}
\frac{-1}{L_{q}} & \frac{1}{L} & 0 & \frac{-1}{R_{S}}
\end{array}\right]^{T}, \quad \mathbf{b}_{g}=\left\lfloor\begin{array}{llll}
\frac{1}{L_{q}} & 0 & 0 & 0
\end{array}\right]^{T}
$$




$$
\mathbf{A}=\left[\begin{array}{cccc}
\frac{-R_{L q}}{L q} & 0 & 0 & 0 \\
0 & \frac{-R}{L} & \frac{-1}{L} & 0 \\
0 & \frac{1}{C} & 0 & 0 \\
1 & -1 & 0 & 0
\end{array}\right], \quad \begin{aligned}
& \mathbf{B}=\left[\begin{array}{llll}
\mathbf{b} & \mathbf{0} & \mathbf{0} & \mathbf{0}
\end{array}\right], \\
& \mathbf{B}_{g}=\left[\begin{array}{llll}
\mathbf{b}_{g} & \mathbf{0} & \mathbf{0} & \mathbf{0}
\end{array}\right]
\end{aligned}
$$

Equation (3) may be written in compact form as:

$$
\dot{\mathbf{x}}=\mathbf{A x}+\mathbf{B} \hat{\mathbf{v}}+\mathbf{B}_{g} \hat{\mathbf{v}}_{g}
$$

where the "hat" operator $\left(^{\wedge}\right)$ denotes

$$
\hat{\mathbf{z}}=\left[\begin{array}{llll}
z(t) & \frac{d z(t)}{d t} & \cdots & \frac{d^{c} z(t)}{d t^{c}}
\end{array}\right]^{T}
$$

Similarly, equation (4) may be rewritten as

$$
f(v(t))=\mathbf{c}^{T} x(t)+d v(t)+d_{g} v_{g}(t)
$$

where $\mathbf{c}^{T}=\left[\begin{array}{llll}0 & 0 & 0 & 1\end{array}\right]$ and $d=d_{g}=0$ for the class E amplifier in Fig. 1.

Note that for this circuit it is straightforward to obtain the equations corresponding to the off state simply by assuming that the resistance, $R_{s}$, has an infinite value.

\section{B. Circuit equations in terms of the control variable}

Certain steps are required to describe the circuit in terms of the control variable and its successive derivatives for each topology. These steps are carried out in the Laplace domain for operational convenience. First, assuming zero initial conditions, state equations (7) and (9) are expressed in the Laplace transformed domain as

$$
\begin{gathered}
s \mathbf{X}(s)=\mathbf{A} \mathbf{X}(s)+\mathbf{b} V(s)+\mathbf{b}_{\mathbf{g}} V_{g}(s) \\
L\{f(v(t))\}=F(s)=\mathbf{c}^{T} \mathbf{X}(s)+\mathbf{d} V(s)+\mathbf{d}_{\mathbf{g}} V_{g}(s)
\end{gathered}
$$

Combining the two expressions above,

$$
F(s)=\left(\mathbf{c}^{T}(s \mathbf{I}-\mathbf{A})^{-1} \mathbf{b}+\mathbf{d}\right) V(s)+\left(\mathbf{c}^{T}(s \mathbf{I}-\mathbf{A})^{-1} \mathbf{b}_{g}+\mathbf{d}_{g}\right) V_{g}(s)
$$

where the terms multiplying $V(s)$ and $V_{g}(s)$ are quotients of polynomials in $s$ that share the same denominator, the polynomial

$$
\tilde{q}(s)=-\operatorname{det}(s \mathbf{I}-\mathbf{A})
$$

where the negative sign has been introduced for convenience. Now, equation (12) may be rewritten as

$$
\tilde{p}(s) V(s)+\tilde{q}(s) F(s)+\tilde{r}(s) V_{g}(s)=0
$$

where $\tilde{p}(s), \tilde{q}(s)$ and $\tilde{r}(s)$ polynomials in $s$ of an order equal to the circuit order $c$, i.e. 4 in this case. Now, defining the vectors of polynomials coefficients,

$$
\begin{aligned}
\tilde{\mathbf{p}} & =\left[\begin{array}{llll}
\tilde{p}_{0} & \tilde{p}_{1} & \ldots & \tilde{p}_{c}
\end{array}\right]^{T} \\
\tilde{\mathbf{q}} & =\left[\begin{array}{llll}
\tilde{q}_{0} & \tilde{q}_{1} & \ldots & \tilde{q}_{c}
\end{array}\right]^{T} \\
\tilde{\mathbf{r}} & =\left[\begin{array}{llll}
\tilde{r}_{0} & \tilde{r}_{1} & \ldots & \tilde{r}_{c}
\end{array}\right]^{T}
\end{aligned}
$$

equation (14) may be written back in the time-domain as:

$$
\tilde{\mathbf{p}}^{T} \hat{\mathbf{v}}(t)+\tilde{\mathbf{q}}^{T} \hat{\mathbf{f}}(v(t))+\tilde{\mathbf{r}}^{T} \hat{\mathbf{v}}_{\mathbf{g}}(t)=0
$$

\section{Discretization}

The next step is to discretize the equations of the form (16) resulting from each topology. The first-order Gear method (Gear-1, also known as backward Euler) approximates the first derivative as [21]:

$$
\dot{v}_{n}=\frac{1}{\Delta}\left(v_{n}-v_{n-1}\right)
$$

where $\Delta$ is the sampling period and the notation

$$
v_{n}=\left.v(t)\right|_{t=n \Delta}
$$

is used.

Successive derivatives may be obtained by the repeated application of (17), yielding:

$$
\hat{\mathbf{v}}_{n}=\left[\begin{array}{c}
v_{n} \\
\dot{v}_{n} \\
\ddot{v}_{n} \\
\vdots
\end{array}\right]=\left[\begin{array}{cccc}
0 & 0 & 0 & 1 \\
0 & 0 & \frac{-1}{\Delta} & \frac{1}{\Delta} \\
0 & \frac{1}{\Delta^{2}} & \frac{-2}{\Delta^{2}} & \frac{1}{\Delta^{2}} \\
\cdot \cdot & & & \vdots
\end{array}\right]\left[\begin{array}{c}
\vdots \\
v_{n-2} \\
v_{n-1} \\
v_{n}
\end{array}\right]=\mathbf{D}_{1} \mathbf{v}_{n}
$$

D1 is defined as the discretization matrix and allows us to compute the present samples of $v(t)$ and their successive time derivatives from the present and past samples of $v(t)$.

Also, we can write

$$
\hat{\mathbf{f}}_{n}=\mathbf{D}_{\mathbf{1}} \mathbf{f}(\mathbf{v})=\mathbf{D}_{\mathbf{1}}\left[\begin{array}{c}
f\left(v_{n-3}\right) \\
f\left(v_{n-2}\right) \\
f\left(v_{n-1}\right) \\
f\left(v_{n}\right)
\end{array}\right]
$$

without having to use the derivative of $f(v)$.

Finally, the discretized equation equivalent to (16) is obtained as:

$$
\mathbf{p}^{T} \mathbf{v}_{n}+\mathbf{q}^{T} \mathbf{f}\left(\mathbf{v}_{n}\right)+\mathbf{r}^{T} \mathbf{v}_{\mathbf{g}_{n}}=0
$$

where 


$$
\begin{aligned}
\mathbf{p}^{T} & =\tilde{\mathbf{p}}^{T} \mathbf{D}_{\mathbf{1}} \\
\mathbf{q}^{T} & =\widetilde{\mathbf{q}}^{T} \mathbf{D}_{\mathbf{1}} \\
\mathbf{r}^{T} & =\tilde{\mathbf{r}}^{T} \mathbf{D}_{\mathbf{1}}
\end{aligned}
$$

Equation (21) can be particularized for each switch state. This equation allows us to compute a new sample of $v(t)$ from the previous ones, i.e. it allows us to integrate the circuit response.

\section{Relation between the control variable and the state variables}

In order to obtain a relationship between the nonlinearity control variable and the state variables, (9) is successively derived and $\dot{\mathbf{x}}$ is substituted from (7). This yields:

$$
\begin{aligned}
& {\left[\begin{array}{c}
\mathbf{c}^{T} \\
\mathbf{c}^{T} \mathbf{A} \\
\vdots \\
\mathbf{c}^{T} \mathbf{A}^{c-1}
\end{array}\right] \mathbf{x}(t)+\left[\begin{array}{cccc}
d & 0 & \cdots & 0 \\
\mathbf{c}^{T} \mathbf{b} & d & & \\
\vdots & & \ddots & \\
\mathbf{c}^{T} \mathbf{A}^{c-2} \mathbf{b} & & & d
\end{array}\right]\left[\begin{array}{c}
v(t) \\
\dot{v}(t) \\
\vdots \\
c-1 \\
v(t)
\end{array}\right]-} \\
& {\left[\begin{array}{cccc}
1 & 0 & \cdots & 0 \\
0 & 1 & & \\
\vdots & & \ddots & \\
0 & & & 1
\end{array}\right]\left[\begin{array}{c}
f(v(t)) \\
\dot{f}(v(t)) \\
\vdots \\
c-1 \\
f(v(t))
\end{array}\right]+} \\
& {\left[\begin{array}{cccc}
d_{g} & 0 & \cdots & 0 \\
\mathbf{c}^{T} \mathbf{b}_{g} & d_{g} & & \\
\vdots & & \ddots & \\
\mathbf{c}^{T} \mathbf{A}^{c-2} \mathbf{b}_{g} & & & d_{g}
\end{array}\right]\left[\begin{array}{c}
v_{g}(t) \\
\dot{v}_{g}(t) \\
\vdots \\
c-1 \\
v_{g}(t)
\end{array}\right]=\mathbf{0}}
\end{aligned}
$$

where $c$ is the circuit order. This equation is rewritten in compact matrix form as

$$
\mathbf{O x}+\mathbf{M} \hat{\mathbf{v}}-\mathbf{I f}(\mathbf{v})+\mathbf{M}_{\mathrm{g}} \hat{\mathbf{v}}_{\mathrm{g}}=\mathbf{0}
$$

Equation (24) establishes a relation between the state variables and the nonlinearity control variable and its successive derivatives. Using the discretization matrix, this equation can be written as:

$$
\mathbf{O x}+\mathbf{M}_{\mathbf{v}} \mathbf{D}_{\mathbf{1}} \mathbf{v}-\mathbf{I D}_{\mathbf{1}} \mathbf{f}(v)+\mathbf{M}_{\mathrm{g}} \mathbf{D}_{\mathbf{1}} \mathbf{v}_{\mathrm{g}}=\mathbf{0}
$$

which is an implicit function that relates the samples of the control variable to the state variables.

From (25), the state variables, $\mathbf{x}$, can be solved for any instant from a number of samples of the control variable equal to the order of the circuit. This is possible if the matrix $\mathbf{O}$, which is also called the observability matrix [25], is invertible. The circuit state is observable from $\mathbf{v}$, or what is equivalent, $\mathbf{O}$ is invertible if there are no independent sub-circuits in the circuit network. A procedure for the case when $\mathbf{O}$ is not invertible is described in [14]
If the state variables are known, (25) can also be solved to obtain the samples of the control variable, $\mathbf{v}$. Therefore, this equation can be used to obtain the state variables from the samples of the control variable or vice versa.

\section{E. Switching Equations}

To initialize (21) after switching, a number of initial samples of the nonlinear element control variable have to be computed from the last samples before switching. We address this process in this subsection.

The process followed is summarized in Fig. 2. First, the vector of samples of the state variables prior to switching, $\mathbf{x}^{-}$, is obtained from the vector of the last samples of $v(t), \mathbf{v}^{-}$, (step no. 1, Fig. 2). Equation (25) is rewritten in the pre-switching topology (denoted with the superscript "-“") as:

$$
\mathbf{x}^{-}=-\left(\mathbf{O}^{-}\right)^{-1}\left[\mathbf{M}^{-} \mathbf{D}_{\mathbf{1}} \mathbf{v}^{-}+\mathbf{M}_{\mathrm{g}}{ }^{-} \mathbf{D}_{\mathbf{1}} \mathbf{v}_{\mathbf{g}}-\mathbf{I D}_{\mathbf{1}} \mathbf{f}\left(\mathbf{v}^{-}\right)\right]
$$

Second, since switching does not alter the state variables, their initial values in the post-switching topology (denoted with the superscript "+") are obtained directly (step no. 2, Fig. 2) as:

$$
\mathbf{x}^{-}=\mathbf{x}^{+}
$$

Third, the initial samples of the control variable are obtained from the vector of state-variables after switching (step no. 3, Fig. 2). Equation (25) is rewritten for this topology as:

$$
\mathbf{O}^{+} \mathbf{x}^{+}+\mathbf{M}^{+} \mathbf{D}_{\mathbf{1}} \mathbf{v}^{+}+\mathbf{M}_{\mathrm{g}}^{+} \mathbf{D}_{\mathbf{1}} \mathbf{v}_{\mathrm{g}}-\mathbf{I D}_{\mathbf{1}} \mathbf{f}\left(\mathbf{v}^{+}\right)=\mathbf{0}
$$

Note that the initial samples of $v(t), \mathbf{v}^{+}$, are the unknowns in this process. These samples coincide in time with the last samples before the switching instant. The resulting switching equations, which relate $\mathbf{v}^{+}$to $\mathbf{v}^{-}$, are obtained by combining (26), (27) and (28)

$$
\begin{aligned}
& {\left[-\mathbf{O}^{+}\left(\mathbf{O}^{-}\right)^{-1} \mathbf{M}^{-} \mathbf{D}_{1}\right] \mathbf{v}^{-}+\left[\mathbf{O}^{+}\left(\mathbf{O}^{-}\right)^{-1} \mathbf{D}_{1}\right] \mathbf{f}\left(\mathbf{v}^{-}\right)+} \\
& {\left[\mathbf{M}^{+} \mathbf{D}_{1}\right] \mathbf{v}^{+}+\left[-\mathbf{D}_{1}\right] \mathbf{f}\left(\mathbf{v}^{+}\right)+} \\
& {\left[\mathbf{M}_{\mathbf{g}}^{+} \mathbf{D}_{\mathbf{1}}-\mathbf{O}^{+}\left(\mathbf{O}^{-}\right)^{-1} \mathbf{M}_{\mathrm{g}}{ }^{-} \mathbf{D}_{1}\right] \mathbf{v}_{\mathbf{g}}=\mathbf{0}}
\end{aligned}
$$

where no superscript - or + is assigned to $\mathbf{v g}$, since there is no distinction between them in any topology.

An equation similar to (29) must be written for each topology change.

\section{$F$. Increasing the discretization order.}

Since with Gear-1 discretization accuracy is only achieved if the time step $\Delta$ is very small, higher-order discretization methods may be used while retaining the overall strategy. The increase in the discretization order significantly reduces the number of samples required for the same accuracy. For the 
Gear-2 discretization scheme [21], the discretization matrix is:

$$
\left[\begin{array}{c}
v_{n} \\
\dot{v}_{n} \\
\ddot{v}_{n} \\
\vdots
\end{array}\right]=\left[\begin{array}{cccccccc}
0 & 0 & 0 & 0 & 0 & 0 & 0 & 1 \\
0 & 0 & 0 & 0 & 0 & \frac{1}{2 \Delta} & \frac{-4}{2 \Delta} & \frac{3}{2 \Delta} \\
0 & 0 & 0 & \frac{1}{4 \Delta^{2}} & \frac{-8}{4 \Delta^{2}} & \frac{22}{4 \Delta^{2}} & \frac{-24}{4 \Delta^{2}} & \frac{9}{4 \Delta^{2}} \\
\cdot & & & & & & & \vdots
\end{array}\right]\left[\begin{array}{c}
\vdots \\
v_{n-6} \\
v_{n-5} \\
v_{n-4} \\
v_{n-3} \\
v_{n-2} \\
v_{n-1} \\
v_{n}
\end{array}\right]
$$

or in compact form

$$
\hat{\mathbf{v}}_{n}=\mathbf{D}_{22} \mathbf{v}_{n}
$$

Equation (16) is discretized yielding an equation similar to (21), making use of (31). On the other hand, substituting this into (24) yields:

$$
\mathbf{O x}+\mathbf{M D}_{22} \mathbf{v}-\mathbf{I D}_{22} \mathbf{f}(\mathbf{v})+\mathbf{M}_{\mathrm{g}} \mathrm{D}_{22} \mathbf{v}_{\mathrm{g}}=\mathbf{0}
$$

This system of $c$ equations can be solved directly for the $c$ state variables if $\mathbf{O}$ is non-singular. However, the control variable cannot be obtained as a function of the state variables, because in that case there are $2 c$ unknowns and only $c$ equations. Therefore, in order to obtain $\mathbf{v}$, an additional linearly independent set of 4 equations is needed.

For this purpose, (24) is derived with respect to time,

$$
\mathbf{O \dot { \mathbf { x } }}+\mathbf{M} \dot{\hat{\mathbf{v}}}+\mathbf{M}_{\mathrm{g}} \dot{\hat{\mathbf{v}}}_{\mathrm{g}}-\dot{\mathbf{I} \mathbf{\hat { \mathbf { f } }}}(\mathbf{v})=\mathbf{0}
$$

Substituting (7) in (33) we obtain

$$
\mathbf{O A x}+\mathbf{O B} \hat{\mathbf{v}}+\mathbf{M} \dot{\hat{\mathbf{v}}}-\dot{\mathbf{I}}(v)+\mathbf{O B} \hat{\mathbf{f}}_{\mathbf{g}}+\mathbf{M}_{\mathbf{g}} \dot{\hat{\mathbf{v}}}_{\mathbf{g}}=\mathbf{0}
$$

an equation that relates the state variables with the control variable and its derivatives up to order $c+1$, represented by the vectors $\hat{\mathbf{v}}$ and $\dot{\hat{\mathbf{v}}}$.

For vectors $\dot{\hat{\mathbf{v}}}, \dot{\hat{\mathbf{f}}}(\mathbf{v})$ and $\dot{\hat{\mathbf{v}}}_{\mathrm{g}}$ a new discretization technique needs to be defined. To obtain this matrix: (i) a first-order approximation of the first derivative is used, and (ii) higher order derivatives are calculated using a second-order approximation. This combination gives rise to the following matrix, D21, [14]:

$$
\left[\begin{array}{c}
\dot{v}_{n} \\
\ddot{v}_{n} \\
\dddot{v}_{n} \\
\vdots
\end{array}\right]=\left[\begin{array}{cccccccc}
0 & 0 & 0 & 0 & 0 & 0 & \frac{-1}{\Delta} & \frac{1}{\Delta} \\
0 & 0 & 0 & 0 & \frac{-1}{2 \Delta^{2}} & \frac{5}{2 \Delta^{2}} & \frac{-7}{2 \Delta^{2}} & \frac{3}{2 \Delta^{2}} \\
0 & 0 & \frac{-1}{4 \Delta^{3}} & \frac{9}{4 \Delta^{3}} & \frac{-30}{4 \Delta^{3}} & \frac{46}{4 \Delta^{3}} & \frac{-33}{4 \Delta^{3}} & \frac{9}{4 \Delta^{3}} \\
. & & & & & & & \vdots
\end{array}\right]\left[\begin{array}{c}
\vdots \\
v_{n-6} \\
v_{n-5} \\
v_{n-4} \\
v_{n-3} \\
v_{n-2} \\
v_{n-1} \\
v_{n}
\end{array}\right]
$$

or in compact form

$$
\dot{\hat{\mathbf{v}}}_{n}=\mathbf{D}_{21} \mathbf{v}_{n}
$$

Now (34) can be discretized using both $\mathbf{D}_{22}$ and $\mathbf{D}_{\mathbf{2 1}}$ :

$$
\begin{gathered}
\text { OAx }+\mathrm{OBD}_{22} v+\mathrm{MD}_{21} \mathrm{v}+\mathrm{OB}_{\mathrm{g}} \mathrm{D}_{22} \mathbf{v}_{\mathrm{g}}+\mathrm{M}_{\mathrm{g}} \mathrm{D}_{21} \mathbf{v}_{\mathrm{g}}- \\
\mathrm{ID}_{21} \mathbf{f}(\mathbf{v})=\mathbf{0}
\end{gathered}
$$

Finally, the result of combining (32) and (37) is a system of $2 c$ equations in the $2 c$ unknowns of the $\mathbf{v}$, from which $\mathbf{v}$ can be solved. As a result, $\mathbf{v}$ can be obtained from $\mathbf{x}$ and vice versa.

The resulting switching equations are obtained as follows. First, state variables are obtained in the pre-switching topology from (32):

$$
\mathbf{x}^{-}=-\left(\mathbf{O}^{-}\right)^{-1}\left[\mathbf{M}^{-} \mathbf{D}_{22} \mathbf{v}^{-}-\mathbf{I D}_{22} \mathbf{f}\left(\mathbf{v}^{-}\right)+\mathbf{M}_{\mathbf{g}}{ }^{-} \mathbf{D}_{\mathbf{2 2}} \mathbf{v}_{\mathbf{g}}\right]
$$

Next, state conservation implies that

$$
\mathbf{x}^{-}=\mathbf{x}^{+}
$$

and finally, the initial samples in the post-switching topology are obtained from (32) and (37):

$$
\begin{aligned}
& \mathbf{O}^{+} \mathbf{x}^{+}+\mathbf{M}^{+} \mathbf{D}_{\mathbf{2 2}} \mathbf{v}^{+}+\mathbf{M}_{\mathrm{g}}^{+} \mathbf{D}_{\mathbf{2 2}} \mathbf{v}_{\mathbf{g}}-\mathbf{I D}_{\mathbf{2 2}} \mathbf{f}\left(\mathbf{v}^{+}\right)=\mathbf{0} \\
& \mathbf{O}^{+} \mathbf{A}^{+} \mathbf{x}^{+}+\mathbf{O}^{+} \mathbf{B}^{+} \mathbf{D}_{\mathbf{2 2}} \mathbf{v}^{+}+\mathbf{M}^{+} \mathbf{D}_{\mathbf{2 1}} \mathbf{v}^{+}+\mathbf{O}^{+} \mathbf{B}_{\mathrm{g}}^{+} \mathbf{D}_{\mathbf{2 2}} \mathbf{v}_{\mathrm{g}}+ \\
& \mathbf{M}_{\mathrm{g}}^{+} \mathbf{D}_{21} \mathbf{v}_{\mathrm{g}}-\mathbf{I D}_{21} \mathbf{f}\left(\mathbf{v}^{+}\right)=\mathbf{0}
\end{aligned}
$$

Combining (38-41) gives:

$$
\begin{aligned}
& {\left[\begin{array}{c}
-\mathbf{O}^{+} \mathbf{A}^{+}\left(\mathbf{O}^{-}\right)^{-1} \mathbf{M}^{-} \mathbf{D}_{22} \\
-\mathbf{O}^{+}\left(\mathbf{O}^{-}\right)^{-1} \mathbf{M}^{-} \mathbf{D}_{22}
\end{array}\right] \mathbf{v}^{-}+\left[\begin{array}{c}
-\mathbf{O}^{+} \mathbf{A}^{+}\left(\mathbf{O}^{-}\right)^{-1} \mathbf{D}_{22} \\
-\mathbf{O}^{+}\left(\mathbf{O}^{-}\right)^{-1} \mathbf{D}_{22}
\end{array}\right] \mathbf{f}\left(\mathbf{v}^{-}\right)+} \\
& +\left[\begin{array}{c}
\mathbf{O}^{+} \mathbf{B}^{+} \mathbf{D}_{22}+\mathbf{M}^{+} \mathbf{D}_{21} \\
\mathbf{M}^{+} \mathbf{D}_{22}
\end{array}\right] \mathbf{v}^{+}-\left[\begin{array}{l}
\mathbf{D}_{21} \\
\mathbf{D}_{\mathbf{2 2}}
\end{array}\right] \mathbf{f}\left(\mathbf{v}^{+}\right)+ \\
& +\left[\begin{array}{c}
\left(\mathbf{O}^{+} \mathbf{B}_{\mathrm{g}}^{+}{ }^{-} \mathbf{O}^{+} \mathbf{A}^{+}\left(\mathbf{O}^{-}\right)^{-1} \mathbf{M}_{\mathrm{g}}^{-}\right) \mathbf{D}_{22}+\mathbf{M}_{\mathrm{g}}^{+} \mathbf{D}_{21} \\
\left(\mathbf{M}_{\mathrm{g}}{ }^{+}-\mathbf{O}^{+}\left(\mathbf{O}^{-}\right)^{-1} \mathbf{M}_{\mathrm{g}}{ }^{-}\right) \mathbf{D}_{22}
\end{array}\right] \mathbf{v}_{\mathrm{g}}=\mathbf{0}
\end{aligned}
$$

which is a system of equations with the same number of 
unknowns, relating $\mathbf{v}^{+}$to $\mathbf{v}^{-}$.

Renaming the terms in this equation allows us to rewrite (42) in compact form as:

$$
\mathbf{S}^{-} \mathbf{v}^{-}+\mathbf{S}_{\mathbf{f}} \mathbf{f}\left(\mathbf{v}^{-}\right)+\mathbf{S}^{+} \mathbf{v}^{+}-\mathbf{D}_{\mathbf{2}} \mathbf{f}\left(\mathbf{v}^{+}\right)+\mathbf{S}_{\mathrm{g}} \mathbf{v}_{\mathrm{g}}=\mathbf{0}
$$

Note that even-higher-order discretization methods can be used following a similar procedure. However, while increasing from Gear-1 to Gear-2 results in significant improvement, extensive tests show that the results obtained with the Gear-2 discretization order are accurate enough and a good compromise between accuracy and complexity. On the other hand, higher discretization orders may cause instability convergence problems that make them inappropriate for use in this method.

\section{G. The Global System of Equations}

Note that equations of type (21) allow successive samples of $v(t)$ to be computed, while equations of type (43) compute the initial values after each switching. Repeatedly combining them allows us to compute the transient response. However, introducing the periodicity assumption, i.e. $v_{N+l}=v_{l}$, where $T=N \Delta$, yields an algebraic formulation directly in the steadystate.

The global system of steady-state equations is built from two types of equations that correspond to discretized differential equations (21) and the switching equations (43) respectively, while the unknowns are all the samples of a period of the control variable in the steady state, including initial samples.

The number of equations in the global system is:

$$
N_{f}=N+n^{O N}+n^{O F F}
$$

where $N$ is the number of real samples in a period, $n^{O N}$ is the number of initial samples in the on state and $n^{\text {OFF }}$ is the number of initial samples in the off state.

The resulting system of equations is made up of $a$ ) matrices built from parts of circulant matrices of the form

$$
\operatorname{circ}\left(\left[\begin{array}{llll}
c_{1} & c_{2} & \cdots & c_{N_{f}}
\end{array}\right]\right)=\left[\begin{array}{cccc}
c_{1} & c_{N_{f}} & \cdots & c_{2} \\
c_{2} & c_{1} & \cdots & c_{3} \\
\vdots & \vdots & \ddots & \\
c_{N_{f}} & c_{N_{f}-1} & & c_{1}
\end{array}\right]
$$

which in turn are obtained from equations of type (21) and $b$ ) switching matrices corresponding to equations of type (43).

By using the notation (45), the circulant matrices

$$
\begin{aligned}
& \mathbf{P}_{r}=\operatorname{circ}\left(\left[\begin{array}{llll}
\mathbf{p}^{T} & 0 & \cdots & 0
\end{array}\right]\right) \\
& \mathbf{Q}_{r}=\operatorname{circ}\left(\left[\begin{array}{llll}
\mathbf{q}^{T} & 0 & \cdots & 0
\end{array}\right]\right) \\
& \mathbf{R}_{r}=\operatorname{circ}\left(\left[\begin{array}{llll}
\mathbf{r}^{T} & 0 & \cdots & 0
\end{array}\right]\right)
\end{aligned}
$$

are defined.

Parts of these matrices must be placed in the correct position depending on the switching instant and switching state.

With this objective, defining

$$
\begin{aligned}
& \eta^{O N}=\operatorname{int}\left(N \frac{t^{O N}}{T}\right) \\
& \eta^{O F F}=\operatorname{int}\left(N \frac{t^{O F F}}{T}\right)
\end{aligned}
$$

the indexes of the first samples corresponding to the on and the off period are computed respectively as:

$$
\begin{aligned}
& \eta_{f}^{\text {ON }}= \begin{cases}\eta^{\text {ON }} & \eta^{\text {ON }}<\eta^{\text {OFF }} \\
\eta^{\text {ON }}+n^{\text {OFF }} & \eta^{\text {ON }}>\eta^{\text {OFF }}\end{cases} \\
& \eta_{f}^{\text {OFF }}= \begin{cases}\eta^{\text {OFF }}+n^{\text {ON }} & \eta^{\text {ON }}<\eta^{\text {OFF }} \\
\eta^{\text {OFF }} & \eta^{\text {ON }}>\eta^{\text {OFF }}\end{cases}
\end{aligned}
$$

Next, a general placing matrix $\mathbf{I}(a, b, c, d)$ of dimensions $N_{f} \mathbf{x}$ $(b+c+d)$ is defined as [14]:

$$
\begin{aligned}
& \mathbf{I}_{i j}= \begin{cases}1 & \begin{array}{l}
\langle a \leq i+1<a+b\rangle_{\bmod N_{f}} \\
\\
j=i+c+1-a
\end{array} \\
0 & \text { Otherwise }\end{cases} \\
& \forall i: 1 \leq i \leq N_{f} \quad \forall j: 1 \leq j \leq b+c+d
\end{aligned}
$$

where $a, b, c, d$ are parameters. Placing matrices allow inserting discretization equations of the type (21) and switching equations of the type (43) into the correct rows and columns of the matrices of the global system of equations, as will be shown next.

First, the placing matrices for the samples in the on and off states are defined respectively as:

$$
\begin{gathered}
\mathbf{I}_{r}^{O N}=\mathbf{I}\left(\begin{array}{l}
\eta_{f}^{O N}+n^{O N}, \eta_{f}^{O F F}-\left(\eta_{f}^{O N}+n^{O N}\right), \\
\eta_{f}^{O N}+n^{O N}-1, N_{f}-\eta_{f}^{O F F}+1
\end{array}\right) \\
\mathbf{I}_{r}^{\text {OFF }}=\mathbf{I}\left(\begin{array}{l}
\eta_{f}^{\text {OFF }}+n^{O F F}, \eta_{f}^{O N}-\left(\eta_{f}^{O F F}+n^{O F F}\right), \\
\eta_{f}^{\text {OFF }}+n^{\text {OFF }}-1, N_{f}-\eta_{f}^{O N}+1
\end{array}\right)
\end{gathered}
$$

In a similar way, the placing matrices for the initial samples in the on and off states are defined respectively as:

$$
\begin{gathered}
\mathbf{I}_{i}^{O N}=\mathbf{I}\left(\eta_{f}^{O N}, n^{O N}, 0,0\right) \\
\mathbf{I}_{i}^{\text {OFF }}=\mathbf{I}\left(\eta_{f}^{O F F}, n^{O F F}, 0,0\right)
\end{gathered}
$$

and the placing matrices for the last samples in the on and off state are defined respectively as: 


$$
\begin{gathered}
\mathbf{I}_{l}^{O N}=\mathbf{I}\left(\eta_{f}^{O F F}-n^{O N}, n^{O N}, 0,0\right) \\
\mathbf{I}_{l}^{\text {OFF }}=\mathbf{I}\left(\eta_{f}^{O N}-n^{O F F}, n^{O F F}, 0,0\right)
\end{gathered}
$$

Finally, the global system of equations may be written as:

$$
\mathbf{P v}+\mathbf{Q f}(\mathbf{v})+\mathbf{R} \mathbf{v}_{\mathrm{g}}=\mathbf{0}
$$

where

$$
\begin{aligned}
& \mathbf{P}=\underbrace{\mathbf{I}_{r}^{O N} \mathbf{P}_{r}^{O N}}_{\text {real }}+\underbrace{\mathbf{I}_{i}^{O N}\left(\mathbf{S}^{O F F^{-}} \mathbf{I}_{l}^{O F F^{T}}+\mathbf{S}^{O N^{+}} \mathbf{I}_{i}^{O N^{T}}\right)}_{\text {initial }}+ \\
& \underbrace{\underbrace{\text { OFF }}_{\text {FF }} \mathbf{P}_{r}^{\text {OFF }}+\underbrace{\mathbf{I}_{i}^{\text {OFF }}\left(\mathbf{S}^{O N^{-}} \mathbf{I}_{l}^{\text {ON }}-\mathbf{S}^{\text {OFF }} \mathbf{I}_{i}^{\text {OFF }}\right.}_{\text {intial }})}_{\text {real }} \\
& \mathbf{Q}=\mathbf{I}_{r}^{O N} \mathbf{Q}_{r}^{O N}+\mathbf{I}_{i}^{O N}\left(\mathbf{S}_{f}^{O F F} \mathbf{I}_{l}^{O F F^{T}}-\mathbf{D}_{\mathbf{2}} \mathbf{I}_{i}^{O N}\right)+ \\
& +\mathbf{I}_{r}^{\text {OFF }} \mathbf{Q}_{r}^{\text {OFF }}+\mathbf{I}_{i}^{\text {OFF }}\left(\mathbf{S}_{f}^{\text {ON }} \mathbf{I}_{l}^{\text {ONT }}-\mathbf{D}_{\mathbf{2}} \mathbf{I}_{i}^{\text {OFF }}\right) \\
& \mathbf{R}=\mathbf{I}_{r}^{O N} \mathbf{R}_{r}^{O N}+\mathbf{I}_{i}^{O N} \mathbf{S}_{g}^{O N} \mathbf{I}_{i}^{O N T}+\mathbf{I}_{r}^{\text {OFF }} \mathbf{R}_{r}^{\text {OFF }}+\mathbf{I}_{i}^{\text {OFF }} \mathbf{S}_{g}^{O F F} \mathbf{I}_{i}^{\text {OFF }}
\end{aligned}
$$

The unknown vector $\mathbf{v}$ contains the initial samples at each switching instant and the samples of a switching period. Vector $\mathbf{v g}$ contains the samples of a switching period of the external excitation.

The nonlinear system of equations (56) can be efficiently solved by trust neighborhood methods [26] based on modifications of Newton's method. Note that in the linear case (56) provides the steady-state response, solving a linear system of equations. This is used as an initial estimation for the nonlinear case.

\section{VERIFICATION}

To verify the proposed modelling technique, a class-E amplifier was designed for a frequency of $6.78 \mathrm{MHz}$. Table I shows the particular values of the circuit elements. The device used is an IXYS DE275-102N06A RF power MOSFET. The device nonlinear output capacitance parameters, $C_{j 0}, V_{b i}$ and $\gamma$, together with the on resistance were extracted using the technique described in [27] from measurements in the switching conditions. Table I shows the circuit parameters of the amplifier. Accurately estimating the device values enables us to design a class-E amplifier which does not need an external shunt capacitance to be added in order to obtain the class E switching conditions.

The described analysis technique is validated against a PSpice simulation of the circuit in Fig. 1, using a nonlinear capacitor model and an ideal switch for the active device. To achieve a reasonable accuracy, the step ceiling parameter in the PSpice simulation was set to $0.1 \mathrm{~ns}$, which resulted in 1540 samples per period, and it was verified that the steady-state had been achieved after $300 \mathrm{RF}$ periods. The result of this simulation is taken as the reference against which the other waveforms are compared.

Fig. 3 and Fig. 4 show the drain voltage waveform and non linear capacitor current respectively computed with the described method, taking only 82 samples per period, and the PSpice simulation taken as a reference. When the number of samples of the described method is doubled, the two waveforms become indistinguishable.

Table II shows a comparison of the relative mean quadratic error of the $v_{C S}(t)$ and $i_{L q}(t)$ waveform when the presented method and PSpice are compared with the reference solution. The number of samples is 100 in both methods. It should be noted that 18 samples are a result of the initial samples. Therefore, there are only 82 samples that can be represented for this method in Fig. 3.

\section{CONCLUSION}

A new method for determining the steady-state periodic response of nonlinear switched circuits has been presented based on the particular case of a Class E amplifier. A step-bystep description of the method is as follows. First, starting from the state equations (7), the differential equation describing the circuit in terms of the control variable (16) is obtained for each topology. This equation is discretized making use of the Gear integration rules (21). A procedure to compute the initial samples required to initialize the discretized equation (21) after each switching instant is described. These switching equations are obtained from the last samples of the control variable before switching, making use of the conservation of state variables during switching. Equations (29) and (43) describe specific results for first and second order Gear discretizations, respectively. Finally, making use of the periodicity assumption, a global system of equations (56) is built from the discretized differential equations and the switching equations for each topology.

As a consequence, the original problem is transformed into a nonlinear algebraic system of equations, in which the unknowns to be determined are the initial samples and the samples of one period of the nonlinearity control variable in steady state. Any other circuit variable is easily obtained from the nonlinearity control variable.

Accuracy is improved using higher order discretization rules keeping a constant time step at every sampling point. This contrasts with conventional time-domain techniques in which a variable sampling grid is usually obtained, with greater sample density in the vicinity of switching. The exact sample after switching, which may be useful information in some circuits, is computed as a byproduct.

The objective of this approach is an increased accuracy in 
relation to the number of samples. The algorithms have been implemented in Matlab with no special emphasis on efficient coding. Even so, the required computing time is comparable to that of other commercially available products, which make use of compiled and carefully optimized engines.

The resulting formulation allows us to compute the sensitivities of the waveform to different circuit parameters very efficiently. Current research is aimed at using this information for circuit optimization.

A comparison of the simulation results of several circuits, and in particular a nonlinear class $\mathrm{E}$ amplifier, with those obtained from PSpice shows excellent agreement, with the added advantage of not having to compute the transient until the steady state is detected.

\section{REFERENCES}

[1] J. Vlach and A. Opal, "Modern CAD Methods for Analysis of Switched Networks", IEEE Transactions on Circuits and Systems-I, 44, 8, 759762 (1997).

[2] F. Yuan and A. Opal, "Computer Methods for Switched Circuits", IEEE Transactions on Circuits and Systems-I, 50, 8, 1013-1024 (2003).

[3] Masatoshi Nakahara, "A Fast Computer Algorithm for Switching Converters", IEEE Transactions on Power Electronics, 12, 1, 180-186 (1997).

[4] K. K. Tse, Henry Shu-hung Chung and S. Y. Hui "Quadratic StateSpace Modeling Technique for Analysis and Simulation of Power Electronic Converters", IEEE Transactions on Power Electronics, 14, 6, 1086-1100 (1999).

[5] F. Guinjoan, J. Calvente, A. Poveda and L. Martínez, "Large-Signal Modeling and Simulation of Switching DC-DC Converters" IEEE Transactions on Power Electronics, 12, 3, 485-494, (1997).

[6] Q. Li and F. Yuan, "Time-Domain Response and Sensitivity of Periodically Switched Nonlinear Circuits", IEEE Transactions on Circuits and Systems-I, 50, 11, 1436-1446 (2003).

[7] N. Femia, G. Spagnuolo and M. Vitelli, "Steady-State Analysis of SoftSwitching Converters", IEEE Transactions on Circuits and Systems-I, 49, 7, 939-954 (2002).

[8] Z. Lizhong and J. Vlach, "Analysis and Steady State of Nonlinear Networks with ideal Switches", IEEE Transactions on Circuits and Systems-I, 42, 4, 212-214 (1995).

[9] David Bedrosian and Jiri Vlach, "An Accelerated Steady-State Method for Networks with Internally Controlled Switches" IEEE Transactions on Circuits and Systems-I, 39, 7, 520-530 (1992).

[10] Jiri Vlach, Jacek M. Wojciechowski and Ajoy Opal "Analysis of Nonlinear Networks with Inconsistent Initial Conditions", IEEE Transactions on Circuits and Systems-I, 42, 4, 195-200 (1995).

[11] B. De Kelper, L. A. Dessaint, K. Al-Haddad and H. Nakra, "A Comprehensive Approach to Fixed-Step Simulation of Switched Circuits", IEEE Transactions on Power Electronics, 17, 2, 216-224 (2002).

[12] Zeljko Mrcarica, Tihomir Ilic and Vanco B. Litovski, "Time-Domain Analysis of Nonlinear Switched Networks with Internally Controlled Switches", IEEE Transactions on Circuits and Systems-I, 46, 3, 373378 (1999).

[13] Pedrag Pejovic and Dragan Maksimovic, "A Method for Fast TimeDomain Simulation of Networks with Switches", IEEE Transactions on Power Electronics, 9, 4, 449-456 (1994).

[14] F. del Águila-López and P. Palà-Schönwälder, "Analysis and Optimization of Nonlinear Switched Circuits by means of Discrete Time Techniques" (in spanish), Ph.D. dissertation, Univ. Politècnica de Catalunya, Barcelona, Spain, 2003.

[15] N. O. Sokal and A. D. Sokal, "Class E, A new class of high-efficiency tuned single-ended switching power amplifiers" IEEE J. Solid-State Circuits, vol. SC-10, 168-176, (1975).

[16] F. H. Raab, "Idealized operation of the Class E tuned power amplifier". IEEE Trans. Circuits Syst., vol CAS-24, 725-735 (1977).
[17] D. J. Kesser and M. K. Kazimierczuk, "Power Losses and Efficiency of Class E Power Amplifier at Any Duty Cycle", IEEE Transactions on Circuits and Systems-I, 51, 9, 1675-1689 (2004).

[18] F. H. Raab, "Effects of Circuit Variations on the Class E Tuned Power Amplifier", IEEE Journal of Solid-State Circuits, vol. SC-13, 239-247, (1978).

[19] P. Molina-Gaudó, A. Mediano-Heredia and J. Navarro-Artigas, "A Contribution to Nonlinear Class-E Amplifier Device Modeling and Parameter Extraction", Ph.D. dissertation, Univ. de Zaragoza, Spain, 2004.

[20] T. Suetsugu and M. K. Kazimierczuk, "Analysis and Design of Class E Amplifier With Shunt Capacitance Composed of Nonlinear and Linear Capacitances", IEEE Transactions on Circuits and Systems-I, 51, 7, 1261-1268 (2004).

[21] P. J. C. Rodrigues, Computer-Aided Analysis of Nonlinear Microwave Circuits, Artech House, inc. (1998).

[22] D. Frey and O. Norman, "An Integral Equation Approach to the Periodic Steady-State Problem in Nonlinear Circuits", IEEE Transactions on Circuits and Systems, CAS-39, 744-755 (1992).

[23] P. Palà-Schönwälder and J. M. Miró-Sans, "A Discrete-Time Approach to the Steady State Analysis and Optimization of Non-Linear Autonomous Circuits", International Journal of Circuit Theory and Applications 23: 297-310 (1995).

[24] J. Bonet, P. Palà, "A Discrete-Time Approach to the Steady-State and Stability Analysis of Distributed Nonlinear Autonomous Circuits", IEEE Transactions on Circuits and Systems- I, 47, 2, 231-236 (2000).

[25] R. C. Dorf, Modern Control Systems, Addison-Wesley, (1989).

[26] J. E. Dennis and R. B. Schnabel, Numerical Methods for Unconstrained Optimization and Nonlinear Equations, Englewood Cliffs, NJ, Prentice Hall (1983).

[27] P. Molina-Gaudó, C. Bernal, A. Mediano: "Exact analysis of a simple class E circuit version for device characterization purposes". Proceedings of the IEEE- Microwave Theory and Techniques Symposium, 3, 1737-1740, (2003).

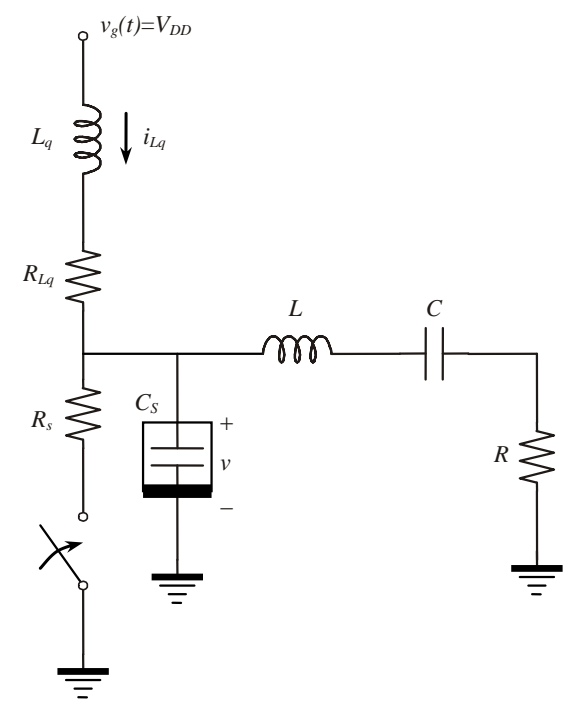

Fig. 1. Class $\mathrm{E}$ amplifier. The switching device is modeled by the ideal switch, $R_{s}$, and the nonlinear capacitance, $C_{s}$. 


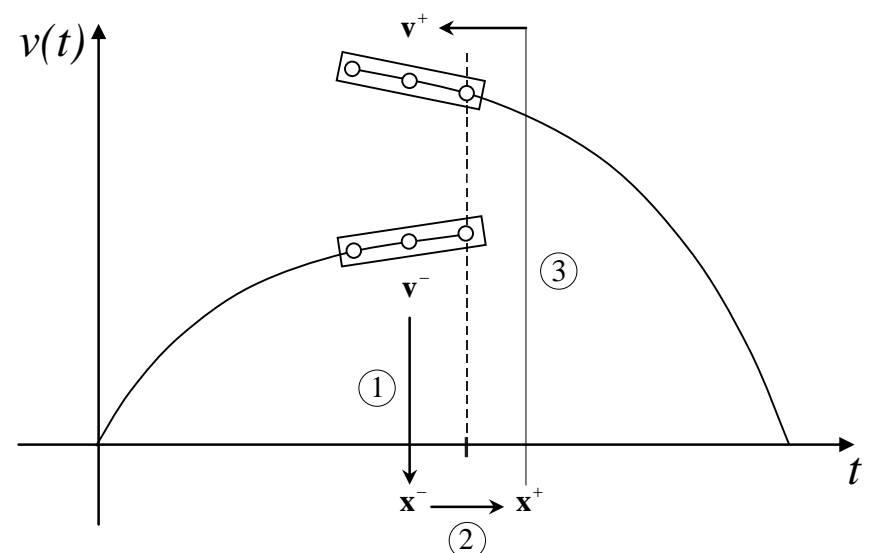

Fig. 2. Computing the initial samples after switching.

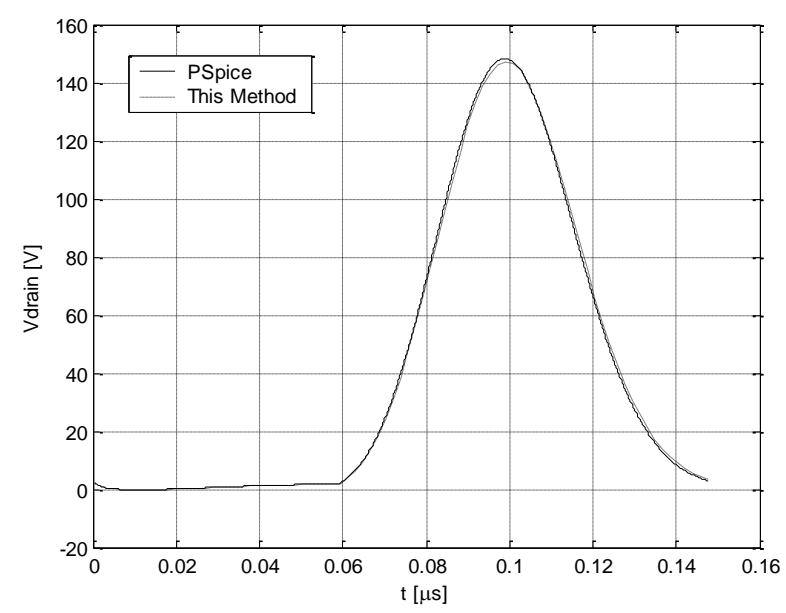

Fig. 3. Comparison of the drain voltage waveform computed with the described method and the PSpice simulation.

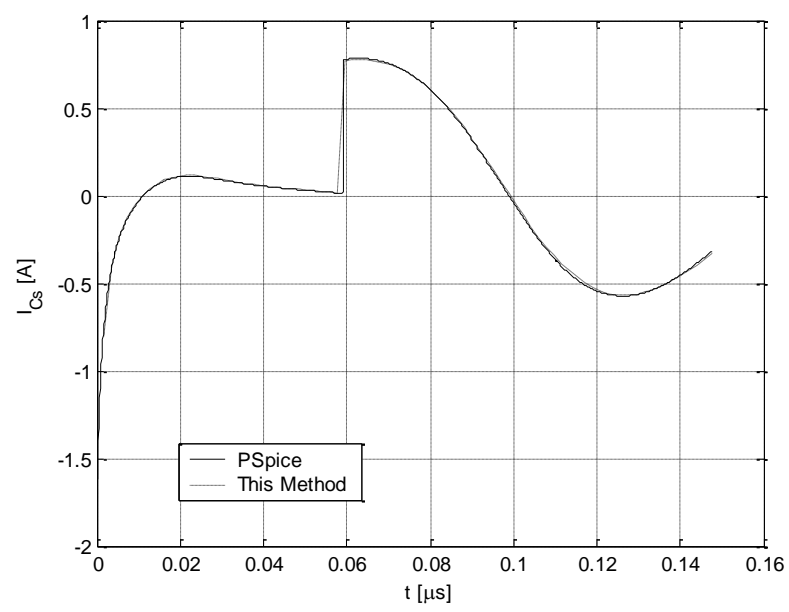

Fig. 4. Comparison of the nonlinear capacitor current waveform computed with the described method and the PSpice simulation.
TABLE I

CLASS-E DESIGN PARAMETERS

\begin{tabular}{ll|ll|ll}
\hline \hline \multicolumn{3}{c|}{ Operation and Linear Devices } & \multicolumn{2}{c}{ Nonlinear Device } \\
\hline \hline $\mathrm{f}$ & $6.78 \mathrm{MHz}$ & $\mathrm{L}_{\mathrm{q}}$ & $97 \mu \mathrm{H}$ & $\mathrm{C}_{\mathrm{j} 0}$ & $2.3 \mathrm{nF}$ \\
$\mathrm{D}$ & 0.4 & $\mathrm{R}_{\mathrm{Lq}}$ & $10 \Omega$ & $\gamma$ & 0.67 \\
$\mathrm{~V}_{\mathrm{DD}}$ & $42 \mathrm{~V}$ & $\mathrm{~L}$ & $6.2 \mu \mathrm{H}$ & $\mathrm{V}_{\mathrm{bi}}$ & $0.71 \mathrm{~V}$ \\
$\mathrm{R}$ & $24 \Omega$ & $\mathrm{C}$ & $138 \mathrm{pF}$ & $\mathrm{R}_{\mathrm{S}}$ & $2.4 \Omega$ \\
\hline \hline
\end{tabular}

TABLE II

RELATIVE ERROR

\begin{tabular}{l|l|l}
\hline \hline & PSpice & This Method \\
\hline \hline$i_{L q}$ & $1.47 \cdot 10^{-2}$ & $5.14 \cdot 10^{-4}$ \\
$i_{C s}$ & $2.85 \cdot 10^{-2}$ & $6.85 \cdot 10^{-4}$ \\
$v_{C s}$ & $7.59 \cdot 10^{-3}$ & $2.96 \cdot 10^{-4}$ \\
\hline \hline
\end{tabular}

Francisco del-Águila-López received his Engineer of Telecommunication and the Ph.D. degrees from the Universitat Politècnica de Catalunya, Barcelona, Spain, in 1996 and 2003, respectively.

Since 1997 he teaches circuit theory, analog electronics, data transmission and telematics. He is currently an Assistant Professor with the Department of Signal Theory and Communications, Technical College of Manresa, Spain.

Dr. del-Águila-López has been involved in several government and industry-funded research projects. His research interests include switched circuits, nonlinear circuits and RF communication circuit design.

Pere Palà-Schönwälder (S'88, M'05) received his Engineer of Telecommunication and the Ph.D. degrees from the Universitat Politècnica de Catalunya, Barcelona, Spain, in 1989 and 1994, respectively.

Since 1990 he teaches circuit theory, analog signal processing, communications electronics and RF design. He is currently an Associate Professor with the Department of Signal Theory and Communications, Technical College of Manresa, Spain.

Dr. Palà-Schönwälder has been the project leader of several government and industry-funded research projects. His research interests include computer-aided circuit design, nonlinear circuits and RF communication circuit design.

Pilar Molina-Gaudó (S'98, M'99, SM'05) received equivalent degree of M.Sc. in telecommunications engineering and Ph.D. degree of electronic engineer from the University of Zaragoza in Spain in 1997 and 2004 respectively. In 1995/96 she was a visiting student at the Technical University of Munich.

Since 2000, she is an Assistant Professor at the Univesrity of Zaragoza researching in the area of power amplifiers for HF/UHF/VHF bands. Her research interests are high frequency, high efficiency switched power amplifiers, in particular modeling the effects of the device in such amplifiers.

Additionally, Dr. Molina-Gaudó is an active volunteer member of the IEEE. She has been a member of the IEEE Women in Engineering Committee from 2001 to 2005 (an area in which she has also done some research), elected Region 8 Student Activities Vice-chair in 2003 and 2004, and thus member of the Region 8 Committee, the R8-OpCom and the IEEE RAB Student Activities Committee for the same period. She is Counselor of the Student Branch at her University. She has been member of the IEEE History Committee in 2004-05 and she is current member of the IEEE New Initiatives Committee as well as the Region 8 sub-committee for Education Activities.

Arturo Mediano (M'98, SM'06) received both his M.Sc. (1990) and his Ph. D. (1997) in Electrical Engineering from the University of Zaragoza, Spain. Since 1992 he has held a teaching professorship with special interests in RF (HF/VHF/UHF) and EMI/EMC design for Telecom and Electrical Engineers. From 1990, he has been involved in design and management responsibilities for research and development projects in the RF field for communications, industry and scientific applications. 
His research interest is focused on high efficiency switching mode RF power amplifiers where he has experience in applications like mobile communication radios, through-earth communication systems, induction heating, plasmas for industrial applications and RFID. He has a solid experience in collaboration with industry (including training and consultancy in $\mathrm{RF}$ and $\mathrm{EMI} / \mathrm{EMC}$ ).

Dr. Mediano is an active member since 1999 of the MTT-17 (HF/VHF/UHF technology) Technical Committee of the Microwave Theory and Techniques Society of the IEEE. 